\title{
Block based texture analysis for iris classification and matching *
}

\author{
Arun Ross \\ West Virginia University \\ arun.ross@mail.wvu.edu
}

\begin{abstract}
The goal of this paper is to analyze the texture of irides and determine if they can be quantitatively measured and assigned into multiple categories. Such an exercise would ensure that irides, like fingerprints, can be partitioned into multiple classes thereby allowing for faster retrieval of identities in large scale biometric systems. In order to facilitate this, a set of 68 statistical features is extracted from the iris texture. These features correspond to the high frequency information associated with anatomical structures in the iris such as crypts, furrows and pigment spots. The statistical features extracted from different blocks in the iris are fused at the feature level and decision level. Experimental analysis using the UPOL database indicates the efficacy of the proposed scheme in (a) clustering iris texture, and (b) assigning an input iris to the correct cluster based on its textural content. The feasibility of using blocks of iris to perform partial iris matching is also investigated.
\end{abstract}

\section{Introduction}

The human iris is a complex textured entity. It begins to form during the third month of the gestation period and the distinctive structures within it are completed by the eighth month [7]. These distinctive structures include pigment frill, collarate, radial furrows, arching ligaments and Fuch's crypts [7], and their agglomerative composition imparts structure and texture to the iris (Figure 1).

The assumed uniqueness of the iris has resulted in its use in the field of biometrics for the purpose of human recognition (verification and identification) [3]. A traditional iris recognition system consists of the following stages: image acquisition, iris segmentation, normalization, feature extraction and matching. An iris recognition system operating in the identification mode typically compares the features extracted from a query image against all the candidate entries in the database in order to determine the best

\footnotetext{
*Project sponsored by US NSF CAREER Award \# IIS 0642554.
}

\author{
Manisha Sam Sunder \\ West Virginia University \\ manishasamsunder@gmail.com
}

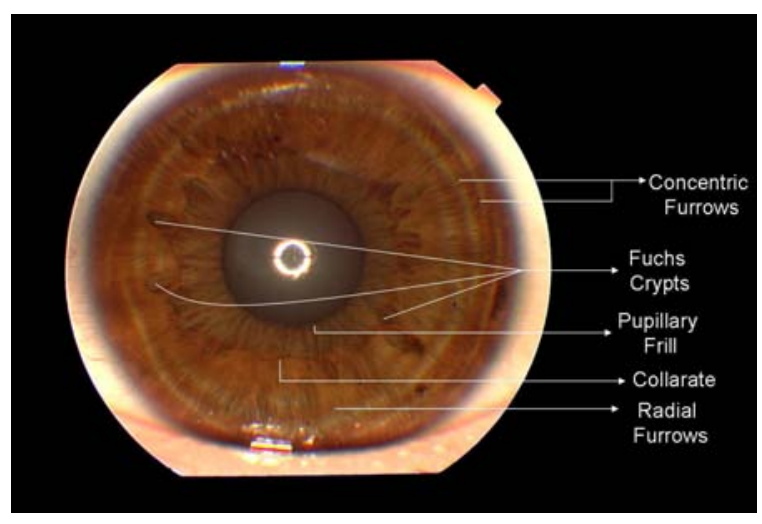

Figure 1. Structures within the iris

match. With the increasing size of iris databases, there is a need to reduce the search space during identification of the query image. Indexing and classification are two ways by which the search space can be reduced during identification. Unlike traditional fingerprint classification systems, there are no standard iris classification systems in place. In the recent literature, there have been some efforts to perform iris indexing and classification. The work by Hao et al [6] introduces the concept of a Beacon Guided Search, where iriscodes are indexed using a unique 32-bit ID that is computed by permuting the iriscode itself. Mukherjee and Ross [9] discuss two schemes for iris indexing. The first scheme, called pre-encoding, uses a texture measure to categorize the iris into multiple bins and organizes them into a tree-like structure to facilitate retrieval; the second scheme, called post-encoding, uses a clustering technique to categorize iriscodes into multiple classes after processing them using first-order statistics. Yu et al [14] perform iris texture classification by computing the fractal dimension on tessellated blocks of the normalized iris, and Qiu et al [11] perform ethnicity classification by analyzing the iris texture using Gabor filters.

The goal of this paper is to study the stochasticity of the iris texture by characterizing it using multiple features and to use the ensuing features to group irides into multiple classes. Thus, it seeks to exploit the textural content of 
the distinctive features composing its anatomy for classification. In order to facilitate this an ensemble of statisticaland signal-based texture characterization schemes is used to define a 68-dimensional feature vector on the normalized iris. For purposes of this study, the UPOL database captured in the visible light is used due to the clarity of the texture present in the component images.

\section{Feature Extraction}

A typical iris recognition system consists of preprocessing steps including segmentation, normalization using Daugman's rubber sheet model, and image enhancement [4]. Segmentation is the process by which the inner and outer boundaries of the iris are localized in an image of the eye. Normalization transforms the points within the iris to polar coordinates $(r, \theta)$, where $r$ represents the radial distance from the iris-pupil boundary to the iris-sclera boundary, and $\theta$ represents the angle subtended by a pixel relative to the horizontal axis. This transformation model accounts for the scaling due to pupil dilations and different diameters of the iris across subjects. The pixel at location $(x, y)$ within the iris image $I$ is transformed to polar coordinates using the following mapping [4]:

$$
I(x(r, \theta), y(r, \theta)) \rightarrow I(r, \theta),
$$

where, $x(r, \theta)$ and $y(r, \theta)$ are pixel positions within the circular disk defined as the linear combination of points on the pupillary boundary $\left(x_{p}(\theta), y_{p}(\theta)\right)$ and limbus boundary $\left(x_{s}(\theta), y_{s}(\theta)\right)$. Thus,

$$
\begin{aligned}
& x(r, \theta)=(1-r) x_{p}(\theta)+r x_{s}(\theta) \\
& y(r, \theta)=(1-r) y_{p}(\theta)+r y_{s}(\theta),
\end{aligned}
$$

The normalized iris images are enhanced using the contrast-limited adaptive histogram equalization (CLAHE) [15] technique. Enhancement involves tessellating the normalized iris into 64 tiles, and subjecting each tile to histogram equalization such that its original histogram distribution matches a uniform flat distribution. Each of the neighboring tiles are bilinearly interpolated to remove the artificially induced boundaries. In order to minimize the effect of noise due to eyelids and eyelashes, only the inner iris region extending from the pupil-iris boundary to half the iris radius is considered for feature extraction. Gentile et al [5] observed that regions close to the pupil and sclera contribute the least to the discriminatory information contained within the iris. The inner half is resized and partitioned to overlapping blocks of size $32 \times 2^{i}$, where $6 \leq i \leq 9$. Figure 2 shows the segmented, normalized and the enhanced iris along with the region of interest used in this work and the iris blocks used for feature extraction.

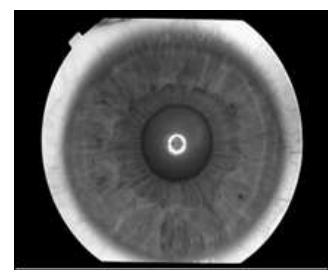

(a) Acquired Iris Image

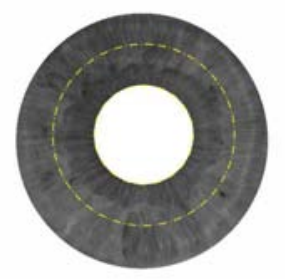

(b) Segmented Iris

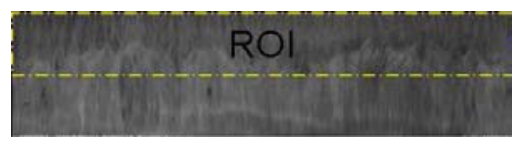

(c) Normalised Iris

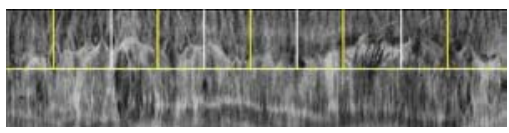

(d) Enhanced Image

Figure 2. The output of the individual pre-processing steps are illustrated here. In (c) the inner iris region used in this work is indicated. (d) illustrates the overlapping blocks (represented here as white and yellow boxes) used for feature extraction.

The agglomeration of structures within the iris impart stochasticity and uniqueness to each eye. In general, textural features can be characterized using statistical, geometrical, model and signal processing based methods [13]. Statistical methods measure the spatial distribution of gray scale values within the image and model the statistical relation between pixels in the image. Thus, statistical features typically correspond to higher order pixel-intensity statistics. Signal processing based methods are based on decomposing an image into frequency and orientation components [13]. Due to the non-deterministic nature of the iris texture, a combination of both the aforementioned methods is used. The statistical features extracted are based on the work by Portilla and Simoncelli [10]. The features are extracted from individual iris blocks each of which is decomposed using complex steerable pyramid at $N=2$ scales and $O=4$ orientations. The steerable pyramid responses are implemented by initially decomposing an image to low pass and high pass residuals $(\mathrm{H})$, and then recursively decomposing the low pass residual of an image into orientation $\left(B_{k}\right)$ and low pass residual bands $(\mathrm{L})$. The filters used are polar separable in Fourier domain and are represented 
as:

$$
\begin{gathered}
L(r, \theta)= \begin{cases}2 \cos \left(\frac{\pi}{2} \log _{2}\left(\frac{4 r}{\pi}\right)\right), & \frac{\pi}{4}<r<\frac{\pi}{2} \\
2, & r \leq \frac{\pi}{4} \\
0, & r \geq \frac{\pi}{2}\end{cases} \\
B_{k}(r, \theta)=H(r) G_{k}(\theta), \quad k \in[0, K-1],
\end{gathered}
$$

where $K$ is the total number of orientations and $H(r)$ and $G_{k}(\theta)$ are the radial and angular parts written as,

$$
\begin{gathered}
H(r)= \begin{cases}\cos \left(\frac{\pi}{2} \log _{2}\left(\frac{2 r}{\pi}\right)\right), & \frac{\pi}{4}<r<\frac{\pi}{2} \\
1, & r \geq \frac{\pi}{2} \\
0, & r \leq \frac{\pi}{4}\end{cases} \\
G(\theta)= \begin{cases}\alpha_{K}\left[\cos \left(\theta-\frac{\pi k}{K}\right)\right]^{K-1}, & \left|\theta-\frac{\pi k}{K}\right|<\frac{\pi}{2} \\
0, & \text { otherwise }\end{cases}
\end{gathered}
$$

Here, $(r, \theta)$ are polar frequency coordinates and $\alpha_{K}=$ $2^{k-1} \frac{(K-1) !}{\sqrt{K[2(K-1)] !}}$. Figure 3 and 4, respectively, illustrate the orientation bands in the frequency domain and the orientation responses on iris blocks of size $64 \times 128$ at $N=2$ scales and $O=4$ orientations.
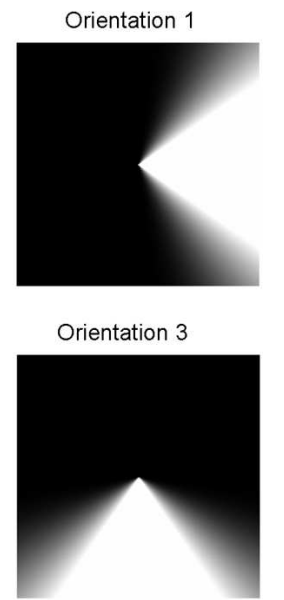

Figure 3. Orientation bands in the frequency domain

The visual texture of the iris can be characterized in terms of its pixel intensity distribution, coarseness, fineness, and regularity due to the presence of periodic or globally oriented structures such as radial furrows, contraction furrows and pigmentation spots. Apart from this, the crypts and furrows at different scales produce oriented edges and corners. The agglomeration of these structures results in the stochasticity of the iris texture and can be captured using statistical features. In the proposed method these statistics

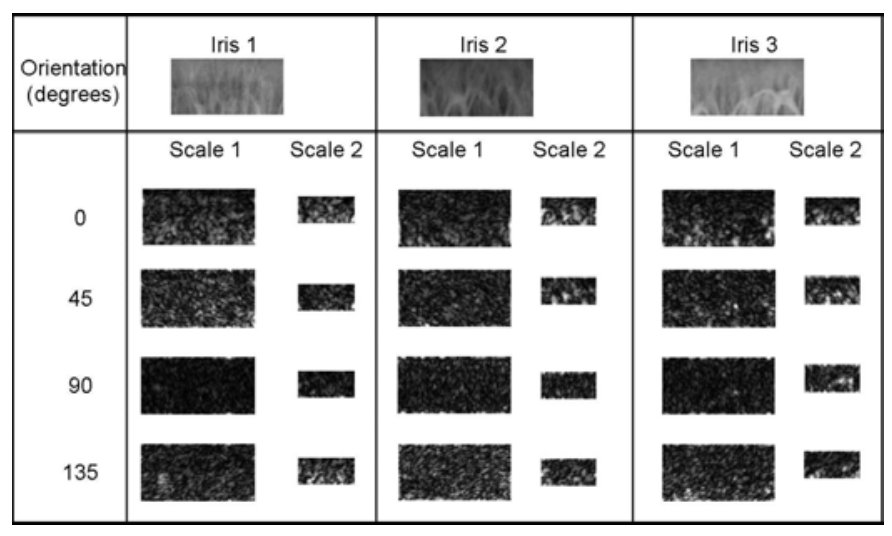

Figure 4. Iris blocks and corresponding responses

are measured from the original iris image as well as the decomposed subbands of the iris.

The statistical features used for modeling the pixel statistics are the minimum, maximum, mean, variance, skewness and kurtosis of pixel-intensity distribution within the iris texture. These features are extracted from the original image as well as from the low pass bands at each scale of the decomposed pyramid. The spectral features which represent periodicity (spectral peaks) and ridges (globally oriented structures) are captured by computing the local autocorrelation of the lowpass residuals at each level of the pyramid decomposition. The high contrast regions oriented at each scale such as edges, bars and corners are captured using the cross correlation of of subband magnitudes with all orientations at the same scale. Given an image $I$ with total number of pixels $|I|$, pixel intensities $x_{i}$ and mean of all pixel intensities $m$, the variance skewness and kurtosis are computed as,

$$
\begin{gathered}
\text { Variance, } \sigma^{2}=\frac{1}{|I|} \sum_{1 \leq i \leq|I|}\left(x_{i}-m\right)^{2}, \\
\text { Skew }=\frac{1}{|I|} \sum_{1 \leq i \leq|I|} \frac{\left(x_{i}-m\right)^{3}}{\sigma^{3}}, \\
\text { Kurtosis }=\frac{1}{|I|} \sum_{1 \leq i \leq|I|} \frac{\left(x_{i}-m\right)^{4}}{\sigma^{4}},
\end{gathered}
$$

For each iris block, all these statistical features are computed, resulting in a feature vector of dimension 68 corresponding to pixel intensity distribution ( 9 features), autocorrelation $\left((N+1)\left(\frac{M^{2}+1}{2}\right)=39\right.$ features, where $M=5$ is the size of the filter $)$ and cross correlation $\left(N \frac{O(O+1)}{2}=20\right.$ features).

\section{Clustering and Classification}

The goal of clustering in the context of this work is to create classes of irides and, subsequently, reduce the search 
space of a query image during identification. This is done by classifying the query image into one of the several predetermined classes and limiting the search to the candidate entries in the assigned class. Figure 5 shows the iris recognition system operating in the classification mode.

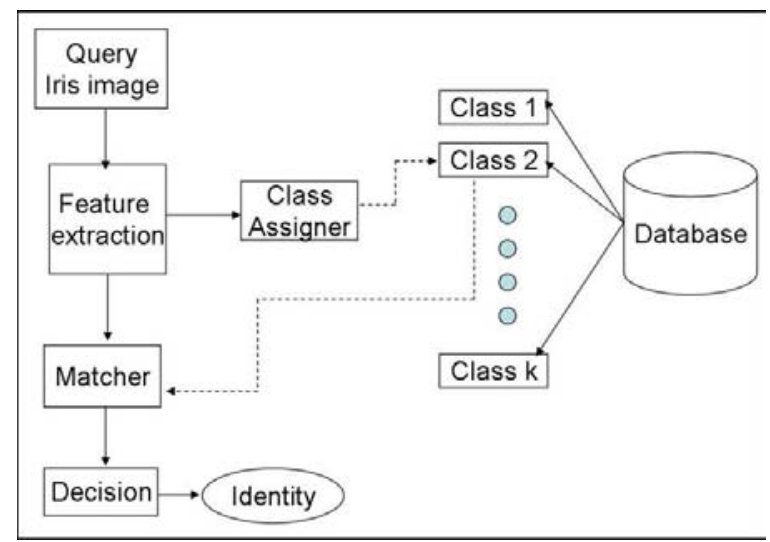

Figure 5. An identification system using classification technique

\subsection{Creating cluster classes}

The Principal Direction Divisive Partitioning [2] technique is adopted to partition the iris database into a predetermined number of clusters. The PDDP algorithm, which is a top-down approach to clustering, is faster than agglomerative clustering algorithms. In order to cluster the iris database, the feature set extracted from a specific block in each iris is used. The database comprised of feature vectors extracted from the iris blocks is recursively partitioned using the principal component analysis thereby making the algorithm divisive. During the process, a hierarchical partition structure is generated until a pre-determined number of clusters is obtained. Each cluster is partitioned based on the measure of cohesiveness. The magnitude of the eigenvalues within a cluster is used as the measure of scatter or non-cohesiveness of a cluster. The higher the scatter value, higher the non-cohesiveness of the cluster which is then chosen to be split into two. Each member feature vector extracted from the iris block (with a dimension of 68) is assigned to one of the two clusters based on its projection on to the principal component. This is repeated until a desired number of clusters is obtained. Figure 6 shows examples of irides in the 5 clusters obtained using PDDP.

A query image is classified by comparing the extracted feature vector (from a block) against each of the cluster centroids and assigning it to the nearest cluster (class).

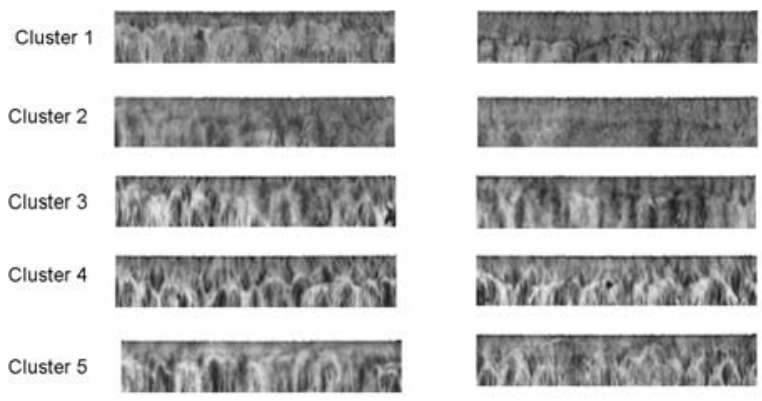

Figure 6. Examples of irides in the 5 clusters.

\section{Database}

The experiments are performed on two datasets of the UPOL $^{1}$ iris database. The UPOL database consists of high resolution iris images in which the iris texture is not unduly occluded due to eyelids and eyelashes. It has $768 \times 57624$ bit RGB color images of the left and right eye of 64 users. The images were acquired using the TOPCON TRC50IA optical device connected to a Sony DXC-950p 3CCD camera. The database consists of 3 samples of each eye resulting in a total of 192 images each of the left and right eyes. The images in the database are subjected to some preprocessing steps where they are first converted to grayscale and the irides manually segmented before normalizing them to size $150 \times 720$ and $64 \times 360$ (low resolution LR - UPOL). The irides normalized to size $64 \times 360$ are used to evaluate the performance of the proposed approach on low resolution images. Figure 7 shows examples of normalized irides generated from the database. This database was chosen due to the clarity of the texture observed on the surface of the iris. As will be shown later, the performance of the proposed classification scheme deteriorates when the iris images do not reveal strong texture. Since the purpose of this work is to understand the textural intricacy of the irides and subsequently cluster them, the UPOL database was used in this work to demonstrate this possibility.

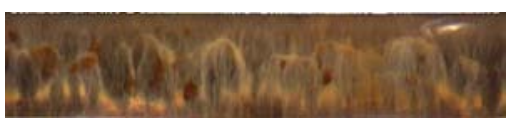

(a) UPOL

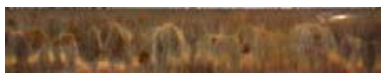

(b) Low-resolution UPOL

Figure 7. Example of normalized iris images in the database

\footnotetext{
${ }^{1}$ http://www.inf.upol.cz/iris/
} 


\section{Experimental setup}

In order to evaluate the potential of using texture features for clustering, the database is split into two sets: training set and test set. Each iris is partitioned into blocks of widths $2^{i}$, where $6 \leq i \leq 9$. The iris blocks are sequentially numbered from 1 to $n$, where $n$ represents the maximum number of blocks obtained with width $w$ from the normalized iris. The features extracted from the irides in the training set are used to obtain the pre-determined cluster classes and their associated centroids. To determine the clustering capability of the statistical textural features extracted, three experiments with different number of clusters $\left(n_{c}=3,5\right)$ are conducted using the test set. The test set is partitioned into the gallery set and the probe set. The gallery set is constructed by either (a) randomly selecting one sample of each iris and assigning it to one of the clusters by computing its distance from each of the cluster centroids, or (b) systematically choosing the first, second or third sample of each iris. The probe set consists of the remaining two samples of each iris and is used for evaluating the classification performance. The classification is deemed to be correct if the probe sample of a subject is assigned to the same class as the corresponding gallery sample. Figure 8 illustrates the experimental set-up. The three experiments performed are summarized below:

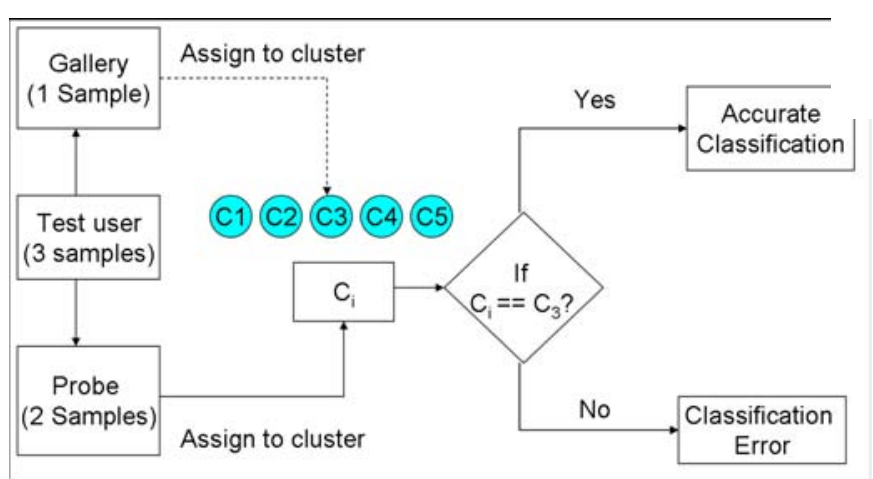

Figure 8. The evaluation protocol adopted in this work

1. Simple classification: Classifying an iris using features extracted from a single block of the iris.

2. Feature level fusion: Classifying an iris by concatenating two feature sets extracted from two different blocks of the same size from the iris.

3. Decision level fusion: Classifying an iris after independently classifying two different blocks within the iris and determining if either of them is assigned to the correct class.

Figure 9 shows the stages involved in the three experiments conducted.

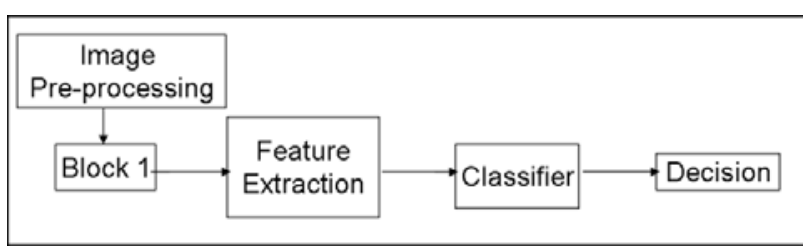

(a) Setup for experiment 1

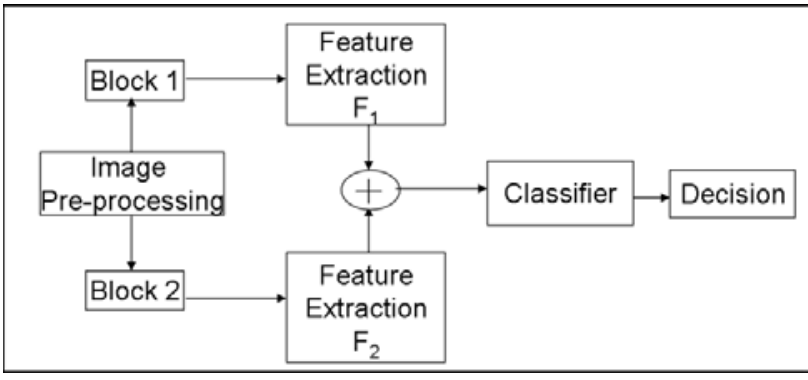

(b) Setup for experiment 2

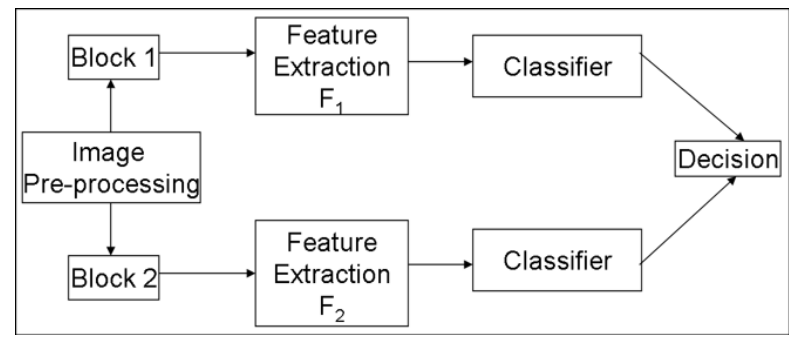

(c) Setup for experiment 3

Figure 9. (a) Setup for experiment 1 using a single iris block. (b) Setup for experiment 2 using two different iris blocks (Feature Level Fusion). (c) Setup for experiment 3 using two different iris blocks (Decision Level Fusion).

\section{Results}

The experiments are conducted using iris blocks with widths $2^{i}$, where $6 \leq i \leq 9$. The classification accuracy is evaluated as the percentage of probe irides that are correctly assigned to the same cluster as that of the corresponding gallery iris. The following tables report the best classification rate for iris blocks of different sizes.

Experiment 1: The features extracted from a single iris block are used for the purpose of evaluation. Tables 1 and 2 show the results of experiment 1 . The distribution of users across the 5 clusters using blocks of width 128 was observed to be $30.47 \%, 23.83 \%, 21.48 \%, 20.70 \%$ and $3.52 \%$, respectively.

Experiment 2: The features extracted from two different iris blocks are concatenated before classification. The results are shown in Tables 3 and 4 .

Experiment 3: The features extracted from two different iris blocks are used independently to classify the iris. Tables 5 and 6 show the results of decision level fusion 
Table 1. Results of Experiment 1 with 3 clusters

\begin{tabular}{|c|c|c|c|c|c|c|c|c|}
\hline & \multicolumn{8}{|c|}{ Width of tessellated blocks } \\
\hline Database & \multicolumn{2}{|c|}{64} & \multicolumn{2}{|c|}{128} & \multicolumn{2}{|c|}{256} & \multicolumn{2}{|c|}{512} \\
\hline & Mean & StD & Mean & StD & Mean & StD & Mean & StD \\
\hline UPOL(L) & 89.84 & 0.40 & $\underline{\mathbf{9 2 . 5 7}}$ & 1.01 & 91.21 & 0.96 & 85.52 & 2.31 \\
\hline UPOL(R) & 92.77 & 0.96 & $\underline{\mathbf{3 3 . 1 6}}$ & 0.55 & 91.21 & 0.55 & 84.76 & 3.45 \\
\hline LR-UPOL(L) & $\underline{\mathbf{9 1 . 0 1}}$ & 1.01 & 90.62 & 0 & 81.83 & 0.96 & - & - \\
\hline LR-UPOL(R) & $\underline{\mathbf{8 8 . 4 7}}$ & 2.59 & 85.54 & 0.61 & 87.30 & 1.78 & - & - \\
\hline
\end{tabular}

Table 2. Results of Experiment 1 with 5 clusters

\begin{tabular}{|c|c|c|c|c|c|c|c|c|}
\hline & \multicolumn{7}{|c|}{ Width of tessellated blocks } \\
\hline Database & \multicolumn{2}{|c|}{64} & \multicolumn{2}{|c|}{128} & 256 & \multicolumn{2}{|c|}{512} \\
\hline & Mean & StD & Mean & StD & Mean & StD & Mean & StD \\
\hline UPOL(L) & $\underline{\mathbf{8 5 . 3 5}}$ & 3.40 & 83.98 & 3.45 & 82.22 & 3.00 & 79.10 & 5.84 \\
\hline UPOL(R) & 85.54 & 2.64 & $\underline{\mathbf{8 8 . 6 7}}$ & 0.20 & 83.00 & 1.78 & 81.44 & 0.55 \\
\hline LR-UPOL(L) & $\underline{\mathbf{8 5 . 1 5}}$ & 2.84 & 81.05 & 2.99 & 75.97 & 2.59 & - & - \\
\hline LR-UPOL(R) & $\underline{\mathbf{8 5 . 3 5}}$ & 0.15 & 81.44 & 0.96 & 79.49 & 3.00 & - & - \\
\hline
\end{tabular}

Table 3. Results of Experiment 2 with 3 clusters

\begin{tabular}{|c|c|c|c|c|c|c|c|c|}
\hline & \multicolumn{9}{|c|}{ Width of tessellated blocks } \\
\hline Database & \multicolumn{2}{|c|}{64} & \multicolumn{2}{|c|}{128} & \multicolumn{2}{|c|}{256} & \multicolumn{2}{|c|}{512} \\
\hline & Mean & StD & Mean & StD & Mean & StD & Mean & StD \\
\hline UPOL(L) & $\underline{\mathbf{9 2 . 9 7}}$ & 1.62 & 91.99 & 1.77 & 85.74 & 0.96 & 85.94 & 3.24 \\
\hline UPOL(R) & $\underline{\mathbf{9 5 . 7 0}}$ & 0.61 & 93.94 & 0.55 & 90.62 & 1.22 & 85.74 & 3.00 \\
\hline LR-UPOL(L) & $\underline{\mathbf{8 9 . 6 5}}$ & 11.54 & 89.61 & 31.06 & 82.42 & 3.44 & - & - \\
\hline LR-UPOL(R) & 88.08 & 0.55 & $\underline{\mathbf{8 8 . 4 7}}$ & 0.55 & 85.54 & 5.08 & - & - \\
\hline
\end{tabular}

Table 4. Results of Experiment 2 with 5 clusters

\begin{tabular}{|c|c|c|c|c|c|c|c|c|}
\hline & \multicolumn{9}{|c|}{ Width of tessellated blocks } \\
\hline Database & \multicolumn{2}{|c|}{64} & \multicolumn{2}{|c|}{128} & \multicolumn{2}{|c|}{256} & \multicolumn{2}{|c|}{512} \\
\hline & Mean & StD & Mean & StD & Mean & StD & Mean & StD \\
\hline UPOL(L) & 86.52 & 0.55 & $\underline{\mathbf{8 6 . 9 1}}$ & 2.58 & 89.68 & 5.28 & 81.05 & 1.77 \\
\hline UPOL(R) & 88.28 & 1.62 & $\underline{\mathbf{8 8 . 4 7}}$ & 0.55 & 80.47 & 0.40 & 76.36 & 0.55 \\
\hline LR-UPOL(L) & $\underline{\mathbf{8 3 . 3 9}}$ & 0.56 & 79.10 & 2.59 & 78.90 & 4.48 & - & - \\
\hline LR-UPOL(R) & $\underline{\mathbf{8 5 . 3 5}}$ & 0.96 & 80.66 & 0.15 & 78.90 & 2.85 & - & - \\
\hline
\end{tabular}

when 3 and 5 clusters are used, respectively.

Table 5. Results of Experiment 3 with 3 clusters

\begin{tabular}{|c|c|c|c|c|c|c|c|c|}
\hline & \multicolumn{7}{|c|}{ Width of tessellated blocks } \\
\hline Database & \multicolumn{2}{|c|}{64} & \multicolumn{2}{|c|}{128} & \multicolumn{2}{|c|}{256} & \multicolumn{2}{c|}{512} \\
\hline & Mean & StD & Mean & StD & Mean & StD & Mean & StD \\
\hline UPOL(L) & $\underline{\mathbf{1 0 0 . 0 0}}$ & 0 & 100.00 & 0 & 98.04 & 0.20 & 94.53 & 0.40 \\
\hline UPOL(R) & $\underline{\mathbf{1 0 0 . 0 0}}$ & 0 & 100.00 & 0 & 99.21 & 0 & 96.48 & 0.20 \\
\hline LR-UPOL(L) & $\underline{\mathbf{9 9 . 4 1}}$ & 0.15 & 99.02 & 0.15 & 95.11 & 0.96 & - & - \\
\hline LR-UPOL(R) & $\underline{\mathbf{1 0 0}}$ & 0 & 99.02 & 0.15 & 96.28 & 2.18 & - & - \\
\hline
\end{tabular}

Table 6. Results of Experiment 3 with 5 clusters

\begin{tabular}{|c|c|c|c|c|c|c|c|c|}
\hline & \multicolumn{9}{|c|}{ Width of tessellated blocks } \\
\hline Database & \multicolumn{2}{|c|}{64} & \multicolumn{2}{|c|}{128} & \multicolumn{2}{|c|}{256} & \multicolumn{2}{|c|}{512} \\
\hline & Mean & StD & Mean & StD & Mean & StD & Mean & StD \\
\hline UPOL(L) & $\underline{\mathbf{9 9 . 0 2}}$ & 0.15 & 97.85 & 0.15 & 94.72 & 0.55 & 87.50 & 1.22 \\
\hline UPOL(R) & $\underline{\mathbf{9 9 . 2 1}}$ & 0.40 & 98.82 & 0.20 & 96.09 & 0.40 & 95.31 & 0.40 \\
\hline LR-UPOL(L) & $\mathbf{9 7 . 4 6}$ & 0.15 & 96.28 & 0.15 & 89.84 & 4.06 & - & - \\
\hline LR-UPOL(R) & $\underline{\mathbf{9 8 . 8 2}}$ & 0.20 & 96.67 & 0.15 & 94.53 & 2.03 & - & - \\
\hline
\end{tabular}

\section{Analysis of experimental results}

Tables 1 and 2 illustrate the results of experiment 1 using 3 and 5 clusters, respectively. Experiment 1 is performed to determine if blocks with varying sizes can be used for the purpose of texture analysis and classification. From the results it is observed that higher classification accuracies are achieved using blocks of smaller widths rather than larger widths. It is also observed that with increasing widths, the discriminability of the features extracted decreases resulting in lower classification accuracy. Furthermore, the classification accuracies are affected by the resolution of the iris. With decreasing resolution, the classification accuracies are observed to decrease in most cases.

To further improve the results of accuracy, feature level fusion and decision level fusion are performed where features from two different iris blocks are used. In feature level fusion, the performance of classification either decreases or remains the same as in Experiment 1. The results show that a simple concatenation of features does not improve the performance and may require feature selection. However, classification via decision level fusion using two independent iris blocks from an iris image shows a large improvement in performance. $100 \%$ classification accuracies are observed for blocks with smaller widths once again illustrating the advantage of using smaller iris blocks for classification. The higher performance obtained, can be attributed to the advantage of using blocks with higher discriminating characteristics rather than using the entire normalized iris for classification. Figure 10 shows an example of an iris image where the classification failed when using blocks of width 256.

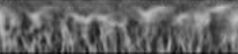

(a) Query image

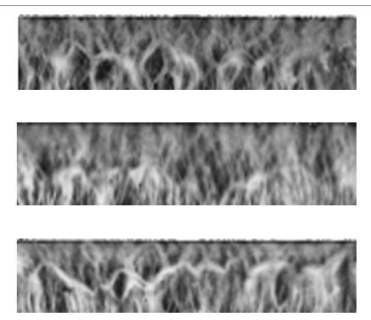

(b) Irides in the class to which (a) belongs.
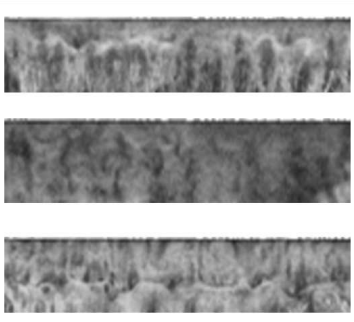

(c) Irides in the class to which (a) was incorrectly assigned.

Figure 10. Example of failed classification. 
The experiments were also conducted on the CASIAIrisV3-Interval ${ }^{2}$ database which includes iris images occluded due to eyelids and eyelashes. It consists of 8-bit gray scale images of size $320 \times 280$ captured using near infrared illumination. The database consists of left and right eye images of 249 users with 1,335 and 1,320 images of the left and right eyes, respectively.

Although the classification accuracies using CASIA-Iris V3-Interval database was not as good as that of the UPOL database, the trend of the results was similar to that of UPOL. The experiments showed that blocks with smaller widths exhibit higher or similar classification accuracies as that obtained using blocks of irides with larger widths. The performance increases from $66 \%$ to $88 \%$ and $55 \%$ to $78 \%$ for Experiment 1 and Experiment 3 with 3 and 5 clusters, respectively. Results once again showed that the method takes advantage of smaller portions of iris which may not be occluded with eyelids and eyelashes for classification. Note that the performance of the classification system on the CASIA database is remarkably inferior. This is due to the low 'clarity' of the texture resident in these iris images. Thus, the proposed technique is relevant for use with iris images exhibiting reasonable textural clarity. Currently, the use of phase information is being pursued in order to devise classification mechanisms for iris databases containing relatively poor quality images.

\section{Block-based recognition}

The above experiments show the feasibility of grouping irides based on features extracted from small blocks of iris. Next, the possibility of using small blocks [12] for matching irides is considered. Specifically, the possibility of matching a partial iris to a complete one is investigated. Recognition is performed using features extracted around keypoints detected on these blocks using the Scale Invariant Feature Transform (SIFT) $[8,1]$. The portions of iris used in this experiment represent $10 \%, 30 \%, 50 \%, 75 \%$ and $100 \%$ of the spatial extent of a normalized iris; the cropped area correspond to the left-most region of the normalized iris. Figure 11 shows examples of the partial irides used in the experiments.

Figure 12 shows the block diagram for partial iris matching using SIFT.

The scale invariant feature transform (SIFT) detects keypoints at multiple scales. The iris block is incrementally convolved with Gaussians to produce images separated with a constant $k$ in scale space. In each octave the difference of Gaussians is obtained by subtracting responses of filtered images in adjacent scales. The extrema points (maxima and minima) in the spatial domain are detected across these Difference-of-Gaussian filtered images. The local ex-

\footnotetext{
${ }^{2} \mathrm{http} / / /$ www.cbsr.ia.ac.cn/IrisDatabase
}

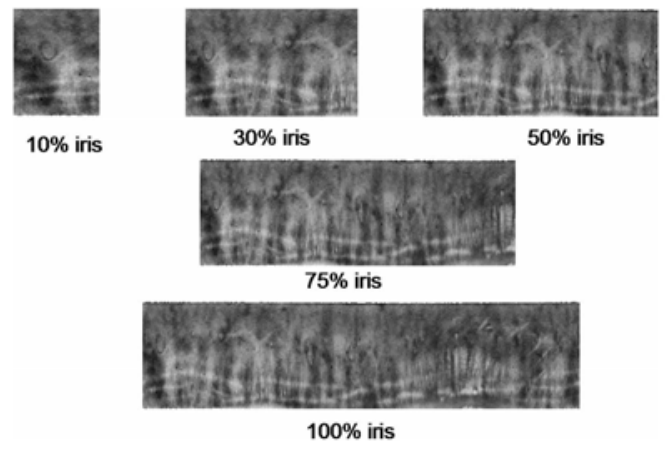

(a) Partial irides

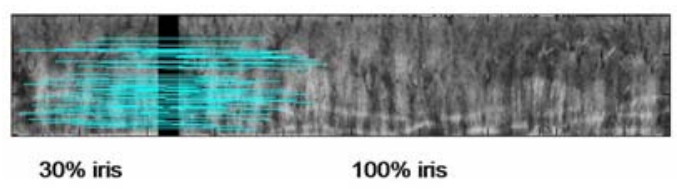

(b) Partial iris matching

Figure 11. (a) Partial iris images representing 10, 30, 50, 75 and 100 percent of the normalized iris. (b) SIFT matching using keypoint descriptors extracted from a partial iris and a complete one.

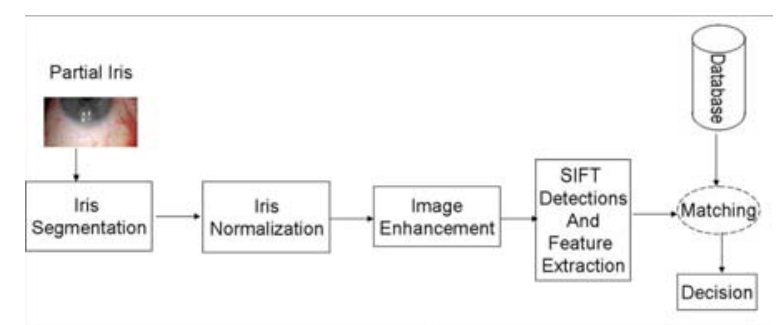

Figure 12. Block diagram to illustrate partial iris recognition using SIFT

trema are determined by checking each pixel for maxima or minima in the neighborhood defined by 8 neighbors in the current image and 9 neighbors each in the images above and below the current image in the Difference-of-Gaussian hierarchy. Orientation histogram is the feature set used to characterize the keypoint and is computed within a descriptor region of size $16 \times 16$ window around the keypoint. The keypoint descriptor is computed as an 8-bin orientation histogram over $4 \times 4$ regions within the descriptor window resulting a 128 dimensional feature vector [8]. The recognition experiment is performed by matching the keypoints detected in the partial irides extracted from normalized irides with the keypoints detected on the complete normalized iris. The match score is the number of keypoints matched between the two iris images. The match between two keypoints is established by computing a cosine similarity score between the features around the keypoints.

The complete UPOL database was used in this experiment involving partial irides - both the left and right eyes 
of all 64 subjects were used to generate the impostor and genuine match scores. The error rates of the recognition experiment using features extracted from portions of the iris are presented in Table 7.

Table 7. Results of partial iris matching

\begin{tabular}{|c|c|}
\hline Portion of iris (\%) & Equal Error Rate (\%) \\
\hline 10 & 0.62 \\
\hline 30 & 0 \\
\hline 50 & 0 \\
\hline 75 & 0 \\
\hline 100 & 0 \\
\hline
\end{tabular}

These results show that using partial irides as small as $30 \%$ of the original normalized iris can result in good matching accuracy. Hence, the iris blocks used for the purpose of classification may also be used for the purpose of recognition.

\section{Summary and future work}

This paper investigated the use of textural information to classify irides into multiple categories. The proposed method extracts statistical features of the iris texture from a block in order to generate a 68-dimensional feature vector. This vector is assigned to one of several pre-determined cluster centroids in order to facilitate iris classification. Experiments show that the resolution of the original iris image and the size of the blocks considered for feature extraction affect the classification accuracy. In addition, the experiments show that performing decision level fusion using two different blocks increases the classification accuracy to $\sim 100 \%$ in the UPOL database. It was also observed that the classification results on the CASIA V3 database were inferior since component images were relatively noisy and lacked textural clarity. As future work, a more detailed analysis on the automated selection of iris blocks with high discriminatory information has to be conducted. Also, the proposed method has to be extended to accommodate poor quality iris images acquired in the near infrared domain. Finally, the design of novel texture descriptors for iris may be necessary due to the stochastic nature of its content.

\section{References}

[1] C. Belcher and Y. Du. Region-based sift approach to iris recognition. Optics and Lasers in Engineering, 47:139-147, 2009.

[2] D. Boley. Principal direction divisive partitioning. Data Mining and Knowledge Discovery, 2(4):325-344, December 1998.

[3] K. W. Bowyer, K. Hollingsworth, and P. J. Flynn. Image understanding for iris biometrics: a survey. Computer Vision and Image Understanding, 110(2):281-307, 2008.
[4] J. Daugman. Probing the uniqueness and randomness of iriscodes: Results from 200 billion iris pair comparisons. Proceedings of the IEEE, 94(11), 2006.

[5] J. E. Gentile, N. Ratha, and J. Connell. SLIC : Short-length iris codes. IEEE 3rd International Conference on Biometrics: Theory, Applications, and Systems (BTAS), September 2009.

[6] F. Hao, J. Daugman, and Z. Piotr. A fast search algorithm for a large fuzzy database. IEEE Transactions on Information Forensics and Security, 3(2):203-212, June 2008.

[7] E. Henderson and C. Margo. Iris melanoma. Archives of Pathology and Laboratory Medicine, 132(2):268-272, February 2008.

[8] D. Lowe. Distinctive image features from scale invariant keypoints. International Journal of Computer Vision, 60(2):91-110, November 2004.

[9] R. Mukherjee and A. Ross. Indexing iris images. In 19th International Conference on Pattern Recognition (ICPR), December 2008.

[10] J. Portilla and E. Simoncelli. A parametric texture model based on joint statistics of complex wavelet coefficients. International Journal of Computer Vision, 40(1):49-71, June 2000.

[11] X. Qiu, Z. Sun, and T. Tan. Global texture analysis of iris images for ethnic classification. Proceedings of International Conference on Biometrics, Lecture Notes in Computer Sciences, 3832:411 - 418, 2005.

[12] J. Thornton, M. Savvides, and B. V. Kumar. A bayesian approach to deformed pattern matching of iris images. IEEE Transactions on Pattern Analysis and Machine Intelligence, 29:596-606, 2007.

[13] M. Tuceryan and A. Jain. Texture analysis. In C. H. Chen, L. F. Pau, and P. S. P. Wang, editors, Handbook Pattern Recognition and Computer Vision, pages 235-276. World Scientific Publishing, 1993.

[14] L. Yu, D. Zhang, K. Wang, and W. Yang. Coarse iris classification using box-counting to estimate fractal dimensions. Pattern Recognition, 38:1791-1798, 2005.

[15] K. Zuiderveld. Contrast limited adaptive histogram equalization. In P. S. Heckbert, editor, Graphics gems IV, pages 474485. Academic Press Professional, San Diego, CA, USA, 1994. 